EPJ Web of Conferences 70, 00084 (2014)

DOI: 10.1051/epjconf/ 20147000084

(C) Owned by the authors, published by EDP Sciences, 2014

\title{
NICA Complex and JINR - status and plans
}

\author{
Vladimir Kekelidze ${ }^{1}$, Alexander Kovalenko ${ }^{1}$, Richard Lednický1,2,a, Viktor Matveev¹, Igor \\ Meshkov ${ }^{1}$, Alexander Sorin ${ }^{1}$, and Grigory Trubnikov ${ }^{1}$ \\ ${ }^{1}$ Joint Institute for Nuclear Research, Dubna, Russia \\ ${ }^{2}$ Institute of Physics AS CR, Prague, Czech Republic
}

\begin{abstract}
One of the main directions of the scientific research at the Joint Institute for Nuclear Research (JINR) in Dubna is the relativistic nuclear and spin physics. The new JINR flagship program in this direction is now realized within the project NICA (Nuclotron-based Ion Collider fAcility). The main goal of the NICA scientific program is an experimental study of hot and dense strongly interacting matter in heavy ion collisions at nucleon-nucleon centre-of-mass energies of 4-11 GeV and at average luminosity of $10^{27} \mathrm{~cm}^{-2} s^{-1}$ for $\mathrm{Au}(79+)$ in the collider mode. In parallel, fixed target experiments at the upgraded JINR superconducting synchrotron Nuclotron are carried out with the extracted beams of various nuclei species up to gold with the momenta up to $13 \mathrm{GeV} / c$ for protons. The program also foresees a study of spin physics with extracted and colliding beams of polarized deuterons and protons at the center-of-mass energies up to $26 \mathrm{GeV}$ for proton collisions. The proposed program allows to search for possible signs of the mixed phase and critical endpoint, and to shed more light on the problem of nucleon spin structure. The survey of the main directions of the JINR scientific research program and general design and construction status of the NICA complex are presented.
\end{abstract}

\section{Introduction}

The Joint Institute for Nuclear Research (JINR) [1] is an international intergovernmental scientific research organisation established in 1956 by eleven founding States and registered with the United Nations in 1957. It is situated in the city of Dubna in Moscow Region of the Russian Federation. The Institute was established to unite the efforts, scientific and material potentials of its Member States in order to study fundamental properties of matter. At present, JINR has 18 Member States that form the supreme governing body of JINR - the Committee of Plenipotentiaries. Participation of six other countries in JINR activities is based on bilateral Agreements signed on the governmental level. One of the main aspects of JINR's activity is its extensive international scientific and technical cooperation: it collaborates with nearly 700 research centres and universities in 64 countries of the world. A bright example is the long-standing collaboration between JINR and CERN, which contributes to a range of theoretical and experimental work in high-energy physics, including experiments at the Large Hadron Collider (LHC) - JINR made a considerable contribution to the design and construction of elements for the LHC machine and its major detectors ATLAS, CMS, ALICE.

a e-mail: lednicky@fzu.cz

This is an Open Access article distributed under the terms of the Creative Commons Attribution License 2.0, which permits unrestricted use, distribution, and reproduction in any medium, provided the original work is properly cited. 
The main fields of JINR's scientific research are theoretical and experimental studies in elementary particle physics, nuclear physics, condensed matter physics and radiation biology. The research policy of JINR is determined by the Scientific Council, which consists of eminent scientists from the Member States as well as famous researchers from other countries, and the European Centre for Nuclear Research (CERN). JINR comprises seven Laboratories, by the scope of scientific activities each being compatible with a large research institution. JINR's staff totals about 5000 people, including more than 1200 scientists, 2000 engineers and technicians. The Institute experimental facilities include: the only in Europe and Asia superconducting accelerator of relativistic nuclei (accelerating particles and heavy ions up to kinetic energy of $6 \mathrm{GeV}$ at $Z / A=1 / 2$ ) - the Nuclotron that is used for the research in the field of relativistic nuclear physics and spin physics, the U-400 and U-400M cyclotrons used for experiments on the synthesis of heavy and exotic nuclei, the unique IBR-2 pulse reactor and resonance neutron source IREN used for nuclear physics research with neutrons and condensed matter studies with the time-of-flight method in the neutron energy range from thermal energies up to hundreds $\mathrm{keV}$, and a $660 \mathrm{MeV}$ proton synchrocyclotron - the phasotron that is mainly used for ray therapy. JINR possesses powerful high productive computing environment that is integrated in the world computer network through high speed communication channels. In 2009 the communication channel "DubnaMoscow" was launched with the initial capacity of $20 \mathrm{Gbit} / \mathrm{s}$ and its gradual increase is planned up to $720 \mathrm{Gbit} / \mathrm{s}$ in 2016. The Central Information Computing Centre of the Institute is actively used to solve the tasks related to experiments at the LHC and other scientific projects that demand large-scale calculations.

The research policy is presently realized within the seven-year plan for the JINR development in 2010-2016 [2]. It envisages concentration of resources for upgrading the accelerator and reactor base of the Institute and integration of its basic facilities into the common system of the European scientific infrastructure. According to this plan, the Nuclotron-NICA project is successfully progressing. It is to become the basis for the new superconducting collider NICA (Nuclotron based Ion Collider fAcility). The heavy ion complex DRIBs-II (Dubna Radioactive Ion Beams) is actively under construction as well. According to the schedule, the spectrometer complex is upgraded of the IBR-2M reactor which is included into the 20-year European strategic programme of research in neutron scattering.

Over five decades of JINR's existence, a wide range of research has been conducted at this centre resulting in a number of achievements. Thus JINR accounts for a half (about 40) of the total number of discoveries in nuclear physics, registered in the former Soviet Union. The decision of the International Committee of Pure and Applied Chemistry to award the name "Dubnium" to element 105 of the Periodic Table can be regarded as a sign of recognition of the outstanding achievements of JINR's staff of researchers in modern physics and chemistry. Among the achievements of the last decade special mentioning deserves synthesis of six new long-life superheavy elements with numbers 113 - 118. These discoveries crowned almost four decades of the effort by experimental physicists in different countries who have been searching for the "stability island" of superheavy nuclei. The two of these elements have been recently awarded the names "Flerovium" (114) after the former director of the Laboratory of Nuclear Reactions Academician G. Flerov and "Livermorium" (116) after the Lawrence Livermore National Laboratory that participated in these discoveries providing the rare isotopes for the target material.

The Joint Institute is a large multidisciplinary international scientific centre, where fundamental nuclear physics research is integrated with the work-out and application of new science-intensive technology and the development of university education in the related fields of science. It provides good conditions for training of specialists of the highest qualification and education of talented young people. Over three decades a branch of Moscow State University has been working in Dubna, the chairs of theoretical and nuclear physics have been opened at the International University of Nature, Soci- 
ety and Man "Dubna". Besides, the JINR University Centre organizes training of the students under the supervision of leading scientists of JINR, taking practice in its laboratories and attending postgraduate courses in physics and mathematics. JINR has actively participated in the implementation of the programme to establish an innovation belt in Dubna. In 2005 it resulted in the establishment of a Special Economic Zone (SEZ) of the technology-innovative type in the territory of Dubna. The specific character of JINR has been revealed in the SEZ appeal: "nuclear physics and information technologies". Among the JINR innovative projects, it is worth to mention the famous nuclear track membranes, the detectors of radiation materials and illicit substances (e.g. explosives or narcotics) or the accelerators for hadron therapy (born in Dubna more than 40 years ago) including the dedicated conformal three-dimensional technology of cancer treatment.

\section{The physics goals of the NICA complex}

The NICA complex is presently under construction at JINR [3]. The research program of the planned experiments at this facility is relevant to understanding the key astrophysical phenomena like the evolution of the early Universe after the Big Bang, formation and structure of neutron stars or the origin of cosmic rays, as well as - to clarifying the physics of relativistic heavy ion collisions and spin phenomena [4-6].

Investigation of hot and dense nuclear matter produced in relativistic heavy ion collisions is a challenging task in modern physics. It provides information on the in-medium properties of hadrons and nuclear matter equation of state, allows for a search of possible manifestations of the deconfinement and/or chiral symmetry restoration phase transitions, mixed phase and critical end-point by scanning various excitation functions in beam energy, atomic number and collision centrality. A number of new phenomena has already been discovered: strong stopping power of colliding nuclei, strong collective flows of secondary particles pointing to a formation of a new form of matter at top RHIC energies behaving like almost ideal and rapidly expanding liquid, constituent quark number scaling of the elliptic flow, a plateau in the apparent temperature at SPS energies and a broadening of transverse momentum distributions at higher energies, irregularities in the beam-energy behavior of the $K / \pi$ ratio, drastic enhancement of multistrange hyperon production, suppression of $J / \psi$ production at SPS energies, essential broadening of the vector meson spectral functions, strong in-medium modification of produced fast hadrons and jets pointing to jet quenching during propagation through the excited nuclear matter, indications on the chiral magnetic effect from three-particle correlations and their disappearance at $\sqrt{s_{N N}}<20 \mathrm{GeV}$.

Different phases of strongly interacting matter are shown in the phase diagram of Fig. 1. One may see that the heavy-ion experiments at RHIC and LHC probe the region of high temperature and low net baryon density where circumstantial evidence has been obtained for a new kind of QCD matter, the strongly interacting quark-gluon plasma (sQGP), existing above a critical temperature $T_{c} \approx 160-170$ $\mathrm{MeV}$ and behaving as an almost ideal liquid. In the other corner of the phase diagram, at a high net baryon density, the matter is deconfined even at a low temperature and, as predicted, correlated quark-antiquark pairs form a color superconductive phase. Such phase may be created in the interior of neutron stars. A fascinating peculiarity is offered in an intermediate region of the phase diagram, where the critical end-point is expected to be located and the phase transition of the excited nuclear matter becomes of the 1st order. The position of the critical end-point is strongly model dependent, the predictions lying in the region of temperature $T_{E} \sim 160-170 \mathrm{MeV}$ and baryon chemical potential $\mu_{E} \sim 200-700 \mathrm{MeV}$. The comprehension of this part of the phase diagram is far from being complete due to the lack of sufficiently accurate data in the corresponding energy domain $\sqrt{s_{N N}}<10 \mathrm{GeV}$. Particularly, the absence of fluctuation and femtoscopic signals of the critical end-point and the onset 


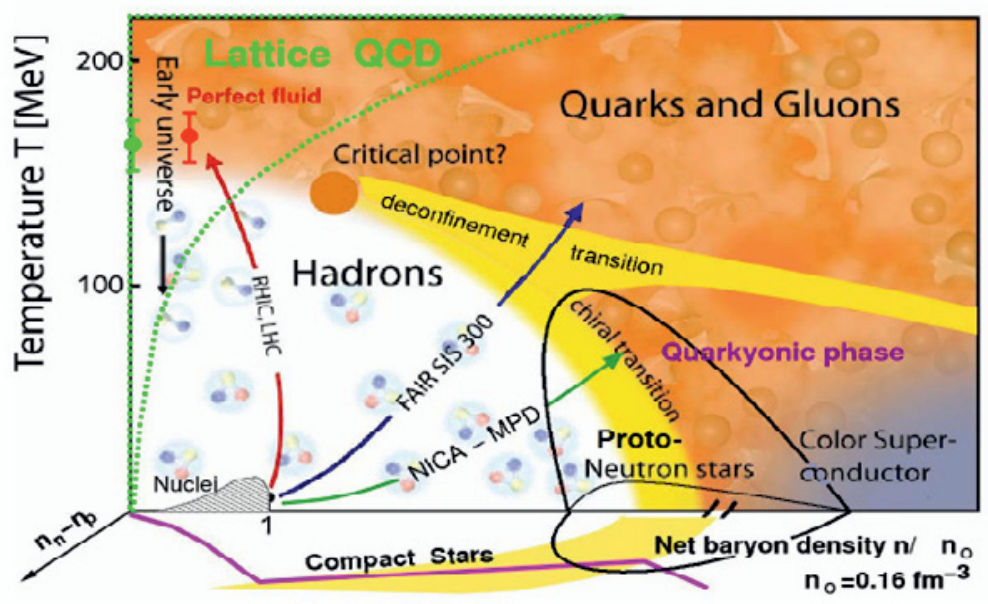

Figure 1. The phase diagram of strongly interacting QCD matter, schematically showing the phase boundaries, critical end-point, and conjectured dynamical trajectories for an expansion stage.

of deconfinement is likely $[6,7]$ due to expected dramatic decrease of the partonic phase in this energy range [8]. The search for these phenomena can thus be successful only in dedicated high statistics and precise experiments.

As a response to this quest, the nuclear and particle physics community initiated formation of an intergovernmental organization to construct a big accelerator complex FAIR (Facility for Antiproton and Ion Research) in Darmstadt with the extracted heavy-ion beams at $E_{l a b}=4-35 \mathrm{AGeV}, \sqrt{s_{N N}}=$ $3-8 \mathrm{GeV}$; the first stage SIS-100 $\left(E_{l a b}<11 \mathrm{AGeV}\right)$ to be available in 2019 and the second one SIS-300 - after 2020. At the BNL-RHIC, the pilot experiments have already been performed at the collider energies reduced from $\sqrt{s_{N N}}=200$ and $130 \mathrm{GeV}$ to $7.7,11.5,19.6,27,39$ and $62.4 \mathrm{GeV}$ despite the loss in the luminosity by 2-3 orders in magnitude at the lowest energies; the low energy scan program at RHIC will continue in 2016-2019 with significantly improved statistics focusing on energies $\sqrt{s_{N N}}<20 \mathrm{GeV}$.

The NICA energy range $\sqrt{s_{N N}}=4-11 \mathrm{GeV}$ is very much lower than those of the RHIC and the LHC, and partly overlaps with the lowest energies available in the RHIC energy scan and the energies of the fixed-target experiments at SPS and FAIR. It sits right on top of the region where the net baryon density is expected to be the highest achievable in terrestrial experiments. In this energy range, the excited nuclear matter occupies the maximum space-time volume in the mixed quark-hadron phase (similar to that of the water-vapor coexistence phase).

Besides the heavy ion beams, the NICA will also provide the polarized proton and deuteron beams up to the c.m.s. energy of $27 \mathrm{GeV}$ for pp collisions with the luminosity higher than $10^{30} \mathrm{~cm}^{-2} \mathrm{~s}^{-1}$. The high intensity and high polarization $(>50 \%)$ will provide a unique possibility for spin physics research, which is of crucial importance for the solution of the nucleon spin problem ("spin puzzle") one of the main tasks of the modern hadron physics. Particularly, a study of the Matveev-MuradyanTavkhelidze-Drell-Yan (MMT-DY) processes, not requiring the input from the poorly known fragmentation functions, can be done in the kinematic region not available in other experiments. 


\section{Superconducting accelerator complex NICA} (Nuclotron based lon Collider fAcility)

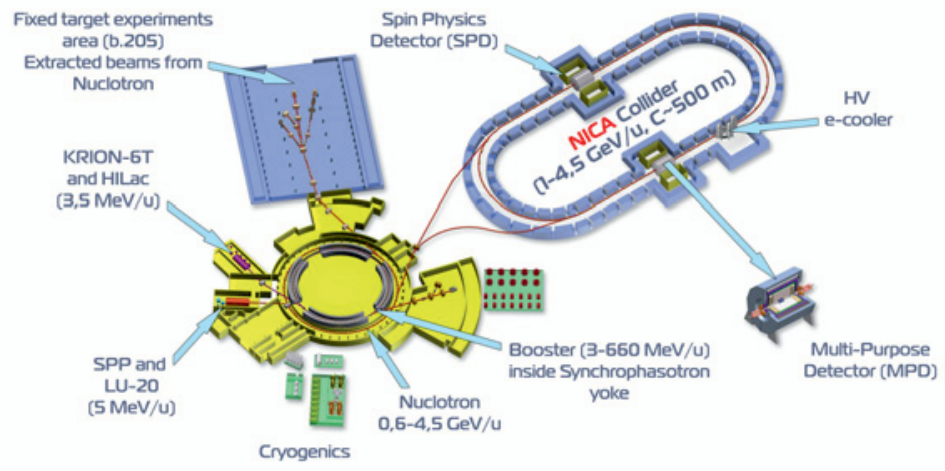

Figure 2. Location of the NICA collider in the JINR accellerator complex area.

\section{NICA layout}

The NICA collider complex is shown on Fig. 2. The construction of this facility is based on the existing buildings and infrastructure of the Synchrophasotron/Nuclotron of the JINR Veksler-Baldin Laboratory of High Energy Physics. The accelerator chain includes heavy-ion and polarized particle sources (KRION-6T and SPP), RFQ injector, heavy- and light-ion linacs (HILac and LU-20), booster synchrotron, Nuclotron and superconducting collider rings. The peak design kinetic energy of gold ions in the collider is $4.5 \mathrm{AGeV}$. Beam cooling and bunching systems are foreseen to achieve the average luminosity of $10^{27} \mathrm{~cm}^{-2} \mathrm{~s}^{-1}$ in the $\mathrm{Au}+\mathrm{Au}$ collisions. The project design presumes the continuation of some of the fixed-target experiments, including those with polarized beams from the Nuclotron. The concept of the NICA project was first presented and discussed at round table discussion in October 2006. It was followed by round table discussion in November 2008 and many other dedicated meetings, including annual meetings of International Machine Advisory Committee. The present project status is available in the Conceptual Design Report [3] and the Technical Design Report is close to its completion. Two interaction points are foreseen at the NICA collider, thus providing a possibility for two detectors to operate simultaneously. The MultiPurpose Detector (MPD) is optimized for the study of properties of hot and dense nuclear matter produced in heavy-ion collisions and the Spin Physics Detector (SPD) - for the study of spin phenomena in the collisions of light polarized ions.

The NICA complex is aimed at the basic science research, yet beams of particles intended for physics experiments are planned to be used for other applications. In particular, JINR has already accumulated essential experience in conducting biomedical research and in performing cancer therapy. The proton and ion beams from the linacs, booster and Nuclotron are well suited for applications and will greatly enhance the JINR capability in many important areas of applied sciences, radiation technology and medicine. 


\section{The detectors: MPD and SPD}

Due to the high complexity of the search for the critical end-point and mixed phase in relativistic heavy ion collisions and large uncertainties in the predicted signals, an accurate scanning of the considered phase diagram domain in the collision energy, impact parameter and system size is utterly needed. In this respect, it is important to provide a uniform detector acceptance over the whole energy range of interest. The operation in the collider mode, as proposed in the NICA project and in the low-energy RHIC program (however, with the luminosity by several orders of magnitude lower than planned in the NICA project), naturally satisfies this demand and has an advantage as compared with the fixed-target mode (SPS, FAIR).

The MPD setup [4] is optimized for a study of the phase diagram of strongly interacting matter produced in heavy-ion collisions at NICA. It has to cover a large phase space, be functional at high interaction rates and comprise high efficiency and excellent particle identification capabilities in a high track multiplicity environment and allow for a controlled selection of the event centralities. The MPD detector concept matching these requirements comprises the central detector and two optional forward spectrometers, the latter covering the pseudorapidity region $2<|\eta|<3$.

The central detector consists of a barrel part and two end-cap trackers located inside the magnetic field. The latter are aimed for precise tracking over pseudorapidity range $1.2<|\eta|<2$. The barrel part covers the pseudorapidity region of $|\eta| \leq 1.2$. It consists of a tracker and particle identification system. The principal tracker is the time projection chamber (TPC) supplemented by the inner tracker (IT) surrounding the interaction region. Both IT (silicon strip detector as a baseline) and TPC have to provide precise track finding, momentum determination, vertex reconstruction and pattern recognition. The energy loss $(\mathrm{dE} / \mathrm{dx})$ measurements in the TPC gas will provide an additional capability for particle identification in low momentum region. The high performance time-of-flight (TOF) system must be able to identify charged hadrons and nuclear clusters in the broad rapidity range and up to total momentum of $2 \mathrm{GeV} / \mathrm{c}$. The fast forward detectors will provide the TOF system with the start signal. In addition, the electromagnetic calorimeter will identify electrons, photons and measure their energy with high precision. Its high granularity together with excellent energy resolution and good timing performances will enhance the overall efficiency and particle identification capabilities of the MPD detector. Particles emitted in very forward/backward directions will be detected by fast forward detectors, beam-beam counters and zero degree calorimeters. They will be used for trigger definition, centrality determination and reconstruction of the position of the interaction point.

The following measurements will be done in the first stage: multiplicity and spectral characteristics of identified hadrons probing entropy production and system temperature at freeze-out; event-byevent fluctuations in multiplicities of various particle species, multiplicity ratios, charges and particle transverse momenta as generic properties of critical phenomena; collective flow effects, particle correlations and femtoscopy with identified particles characterizing collective phenomena and space-time evolution of the excited matter. In the second stage, the electromagnetic probes (photons and dileptons) will be measured.

The NICA facility will also give unique possibilities for spin physics; for running in polarized beam mode the collider will be equipped with the necessary set of spin rotators ("siberian snakes"). For spin physics studies, besides the MPD setup (optimized for a study of multiparticle production), the dedicated SPD setup [5] at the second interaction point is designed similar to the PAX setup at FAIR. It assumes nearly $4 \pi$ acceptance, minimal radiation length to provide an effective detection of lepton pairs and a good angular resolution to allow for a measurement of azimuthal spin asymmetries in a wide kinematic region. The basic SPD parts are: a toroid magnet system with the integrated field of $\sim 0.4 \mathrm{~T} \cdot \mathrm{m}$, inner tracker (Silicon or MicroMega), main tracker (drift chambers or straw tubes), 
Cherenkov counter, electromagnetic calorimeter, trigger counters and EndCap detectors. Also considered is the possibility of so-called beam-dump muon detector.

The following measurements with MPD and SPD are assumed: MMT-DY and $J / \psi$ production processes with longitudinally and transversally polarized proton and deuteron beams for the extraction of unknown or poorly known parton distribution functions; spin effects in baryon, meson, photon and dilepton production including: - $\Lambda$ polarization as a probe of matter isotropization, - correlation of $\Lambda$ polarization with charge separation as a complementary signal of CP-violation in dense matter, - transverse handness as a probe of collective orbital angular momentum, - tensor polarization of dileptons as a probe of matter formation; spin effects in various exclusive reactions and diffractive processes; spin-dependent cross sections, helicity amplitudes and double spin asymmetries (Krisch effect) in elastic reactions; spectroscopy of quarkonia; polarimetry.

\section{Conclusions}

The JINR research policy is being realized within the seven-year plan for the JINR development in 2010-2016. Particularly, it assumes concentration of resources for upgrading its accelerator and reactor basic facilities: the modernization of Nuclotron - the superconducting ion synchrotron and construction on its basis of the new superconducting ion collider NICA to study very important unsolved problems of the physics of strongly interacting matter and spin phenomena, the construction of heavy ion complex DRIBs-II for experiments on the synthesis of heavy and exotic nuclei and the upgrade of the spectrometer complex of the IBR-2 pulse reactor for nuclear physics research with neutrons and condensed matter studies with the time-of-flight method. The design and organizational work on the project of NICA complex started in 2006, and its realization assumes several stages: upgrade of the Nuclotron facility, preparation of the NICA technical design report, start of the prototyping of the NICA, MPD and SPD elements (2007-2012); design, construction and assembling (2012-2016); commissioning (2017). Worldwide cooperation is anticipated at all stages of the project as well as in the elaboration of the scientific program.

\section{References}

[1] The JINR web page, http://www.jinr.ru.

[2] The seven-year plan for the JINR development in 2010-2016, http://www.jinr.ru/7yearplan/Seven_Year_Plan-eng_fin.pdf

[3] N. Agapov et al. (NICA Collaboration), Design and Construction of Nuclotron-based Ion Collider fAcility (NICA), Conceptual Design Report, Dubna, 2008; http://nica.jinr.ru.

[4] Kh.U. Abraamyan et al. (MPD Collaboration), The MultiPurpose Detector - MPD to study Heavy Ion Collisions at NICA (Conceptual Design Report), Dubna 2011, http://nica.jinr.ru; Nucl. Instrum. Meth. A 628, (2011) 99.

[5] A. Efremov et al. (SPD Collaboration), The Spin Physics Detector - SPD to study spin structure of the nucleon and polarization effects at NICA (Conceptual Design Report), Dubna 2011, http://nica.jinr.ru/files/Spin_program/spd_cdr.htm.

[6] NICA White Paper, http://theor.jinr.ru/twiki-cgi/view/NICA/NICAWhitePaper.

[7] R. Lednicky, Nucl. Phys. Proc. Suppl. 198, (2010) 43.

[8] W. Cassing and E. Bratkovskaya, Nucl. Phys. A 831, (2009) 215. 